\title{
Local muscle metabolic demand induced by neuromuscular electrical stimulation and voluntary contractions at different force levels: a NIRS study
}

\author{
Makii Muthalib (1,2), Graham Kerr (3), Kazunori Nosaka (2), Stephane Perrey (1) \\ (1) EuroMov, University of Montpellier, Montpellier, France; (2) School of Medical and \\ Health Sciences, Edith Cowan University, Joondalup, Australia; (3) IHBI, Queensland \\ University of Technology, Brisbane, Australia \\ This article is distributed under the terms of the Creative Commons Attribution Noncommercial License (CC BY-NC 4.0) which \\ permits any noncommercial use, distribution, and reproduction in any medium, provided the original author(s) and source are credited.
}

\begin{abstract}
Functional Muscle metabolic demand during contractions evoked by neuromuscular electrical stimulation (NMES) has been consistently documented to be greater than voluntary contractions (VOL) at the same force level (10-50\% maximal voluntary contraction-MVC). However, we have shown using a near-infrared spectroscopy (NIRS) technique that local muscle metabolic demand is similar between NMES and VOL performed at MVC levels, thus controversy exists. This study therefore compared biceps brachii muscle metabolic demand (tissue oxygenation index-TOI and total hemoglobin volume-tHb) during a 10 s isometric contraction of the elbow flexors between NMES (stimulation frequency of $30 \mathrm{~Hz}$ and current level to evoke 30\% MVC) and VOL at 30\% MVC (VOL-30\%MVC) and MVC (VOL-MVC) level in 8 healthy men (23-33-y). Greater changes in TOI and tHb induced by NMES than VOL-30\%MVC confirm previous studies of a greater local metabolic demand for NMES than VOL at the same force level. The same TOI and tHb changes for NMES and VOL-MVC suggest that local muscle metabolic demand and intramuscular pressure were similar between conditions. In conclusion, these findings indicate that NMES induce a similar local muscle metabolic demand as that of maximal VOL.
\end{abstract}

Key Words: NMES, NIRS, Muscle oxygenation, hemodynamics, muscle activation, voluntary contractions

Eur J Transl Myol 201626 (2) 169-174

\begin{abstract}
Neuromuscular electrical stimulation (NMES) has been shown to be effective for improving muscle strength and endurance in healthy and impaired individuals., However a common effect of NMES-evoked contractions is that fatigue of the stimulated muscles occurs relatively quickly as compared with voluntary (VOL) muscle contractions at a similar force level. ${ }^{3}$ Therefore, it is important to understand the metabolic responses during NMES in comparison to VOL for the development of appropriate exercise and/or rehabilitation training programs. Isometric contractions evoked by NMES induce greater systemic and local muscle metabolic demand than VOL isometric contractions at the same force level (10-50\% of maximal voluntary contraction-MVC), ${ }^{4-8}$ which is suggested to reflect different motor unit recruitment patterns between NMES and VOL. ${ }^{3,9}$ Koistra et al. ${ }^{10}$ utilized near-infrared spectroscopy (NIRS) to compare between NMES (10-s) and maximal VOL (15-s) sustained isometric
\end{abstract}

contractions of the knee extensors for changes in local quadriceps muscle metabolic demand. To ensure maximal activation of the quadriceps muscle in both conditions, VOL was performed at MVC intensity and NMES at maximal stimulation current (70-195 mA) and frequency $(50-75 \mathrm{~Hz})$. The results indicated a similar local muscle metabolic demand during NMES and VOL at MVC intensity, and suggested that this represents similar muscle activation in the region of muscle volume investigated by NIRS. However, a limitation of the study was that there was no direct comparison of NMES and VOL at the same absolute force level. Furthermore, it is still not clear if it is necessary for NMES to activate the muscle at the maximal current intensity or if a lower stimulation frequency $(30 \mathrm{~Hz})$ may be able to achieve a similar muscle metabolic demand as that compared to maximal VOL. Therefore, the purpose of the present study was to utilize NIRS to compare local biceps brachii metabolic demand during a sustained (10-s) isometric 


\section{Muscle metabolic demand by NMES or voluntary contractions at different force levels}

Eur J Transl Myol 26 (2) 169-174

contraction of the elbow flexors between NMES (stimulation frequency of $30 \mathrm{~Hz}$ and current intensity to evoke 30\% MVC) and VOL at the same (VOL$30 \% \mathrm{MVC}$ ) and maximal (VOL-MVC) force level. We hypothesized that NIRS-derived muscle metabolic demand would be greater for NMES than VOL at the same force level (30\% MVC), but similar when NMES was compared to VOL at maximal force level.

\section{Materials and Methods}

\section{Subjects}

Eight healthy men participated in this study, and their mean $( \pm \mathrm{SD})$ age, height, and body mass were $29.4 \pm 8.2$ yrs, $176.0 \pm 5.0 \mathrm{~cm}, 78.1 \pm 9.1 \mathrm{~kg}$, respectively. The study was approved by the local Human Research Ethics Committee in accordance with the Declaration of Helsinki.

\section{Experimental Setup}

Subjects were seated on a preacher arm curl bench, securing the shoulder angle at $45^{\circ}$ flexion with a supinated forearm position and an elbow joint angle of $90^{\circ}$. The elbow joint was aligned with the axis of rotation of a Cybex 6000 isokinetic dynamometer (HUMAC-2004, Computer Sports Medicine Inc., USA). Torque signals were collected onto a data acquisition system (PowerLab, ADInstruments, Australia) at a sampling rate of $200 \mathrm{~Hz}$, and real-time visual feedback of torque signals were displayed on a computer monitor.

\section{Experimental Protocol}

All subjects performed NMES and VOL with the same arm. Part of subjects used their dominant arm and others used their non-dominant arm, which was randomized and counterbalanced among subjects. Subjects initially performed two 3-s MVCs with a $45 \mathrm{~s}$ rest between contractions, and the peak torque of the two contractions was used for further analysis. In order to determine the current intensity to evoke $30 \%$ MVC and to accommodate the subjects to NMES, the current amplitude was gradually ramped up in $10 \mathrm{~s}$ to $10 \% \mathrm{MVC}$ and after a $60 \mathrm{~s}$ rest period the current was further gradually ramped up in $10 \mathrm{~s}$ to evoke $30 \% \mathrm{MVC}$, which was then followed by at least $120 \mathrm{~s}$ rest period. $30 \%$ MVC force level was chosen to compare NMES and VOL at the same absolute force level. In order to evoke $30 \%$ MVC using NMES, current intensity was $47 \pm 3$ $\mathrm{mA}$, which corresponded to $\sim 80 \%$ maximal NMESevoked torque output. After the preliminary NMES and VOL contractions, a 10-s isometric contraction was evoked at $30 \% \mathrm{MVC}$ at the current intensity determined previously (ramp up 0.5-s, ramp down 0-s), and the subjects were then allowed $100 \mathrm{~s}$ rest period. Subjects then voluntarily performed one 10-s isometric contraction at 30\% MVC (VOL-30\%MVC) followed 90 s later by one 10-s MVC (VOL-MVC).

\section{Neuromuscular Electrical Stimulation}

A portable stimulator (Compex2, Medicompex SA, Switzerland) was used to deliver biphasic symmetric rectangular pulses with a frequency of $30 \mathrm{~Hz}$ and pulse duration of $200 \mu \mathrm{s}$. Two self-adhesive surface electrodes of $25 \mathrm{~cm}^{2}(5 \mathrm{~cm} \times 5 \mathrm{~cm})$ were placed along the mid-belly of the biceps brachii and brachioradialis muscles. A dispersive electrode of $50 \mathrm{~cm}^{2}(5 \mathrm{~cm} \times 10$ $\mathrm{cm})$ was placed on the elbow crease.

\section{Near-Infrared Spectroscopy}

The NIRS measurements were undertaken by a NIRO200 oximeter (Hamamatsu Photonics, Japan). The optical probe (consisting of one emitter and one detector $4 \mathrm{~cm}$ apart) was firmly attached to the skin at the mid-belly of the biceps brachii muscle, parallel to the major axis of the arm by a double-sided adhesive tape, which ensured no sliding of the probe on the skin. The optical probe was covered by a soft black cloth to prevent extraneous light influencing the NIRS measurements. NIRS measures the $\mathrm{O}_{2}$ dependent absorption of hemoglobin $(\mathrm{Hb})$ in the muscle microcirculation blood vessels (i.e., arterioles, capillaries, and venules) and myoglobin in the muscle cytoplasm. ${ }^{11}$ The muscle utilization of the oxidative metabolic system is represented by NIRS as concentration changes in oxy-Hb $\left(\mathrm{O}^{2} \mathrm{Hb}\right)$, deoxy- $\mathrm{Hb}$ $(\mathrm{HHb})$, total- $\mathrm{Hb}(\mathrm{tHb})$, and an absolute measure of muscle $\mathrm{O}_{2} \mathrm{Hb}$ saturation ( $\mathrm{TOI}=\mathrm{O} 2 \mathrm{Hb} / \mathrm{tHb} \times 100$ ). The theoretical basis, application and limitations of NIRS in health and disease have been previously reviewed. ${ }^{11,12}$ NIRS signals were sampled at $6 \mathrm{~Hz}$ by the NIRO-200 and collected simultaneously with torque data onto the PowerLab system and stored on the computer for later analysis using the Chart data analysis software. Subcutaneous adipose tissue thickness has been reported to affect NIRS measurement sensitivity, therefore a B-mode ultrasound apparatus fitted with a $7.5-\mathrm{MHz}$ linear probe (SSD-1000, Aloka Co. Ltd., Tokyo, Japan) was used to measure the subcutaneous fat layer thickness of the area over which the optical probe unit was placed. Considering that the adipose tissue thickness was relatively low $(3.0 \pm 0.3 \mathrm{~mm})$, and the penetration depth of the NIRS signal is almost half $(2 \mathrm{~cm})$ of the emitter-detector separation $(4 \mathrm{~cm}$ in the optical probe of the NIRO-200), the changes in TOI and $\mathrm{tHb}$ reflected mainly the metabolic and hemodynamic changes, respectively, of the superficial muscle volume of the biceps brachii.

\section{Data Analysis}

The following torque parameters were considered for the three contraction conditions: 1) mean torque (Torquemean) and 2) torque-time integral (TorqueIntegral), which was determined over the contraction duration. Since no significant difference $(\mathrm{P}>0.05)$ was evident between the three contraction 


\section{Muscle metabolic demand by NMES or voluntary contractions at different force levels}

Eur J Transl Myol 26 (2) 169-174

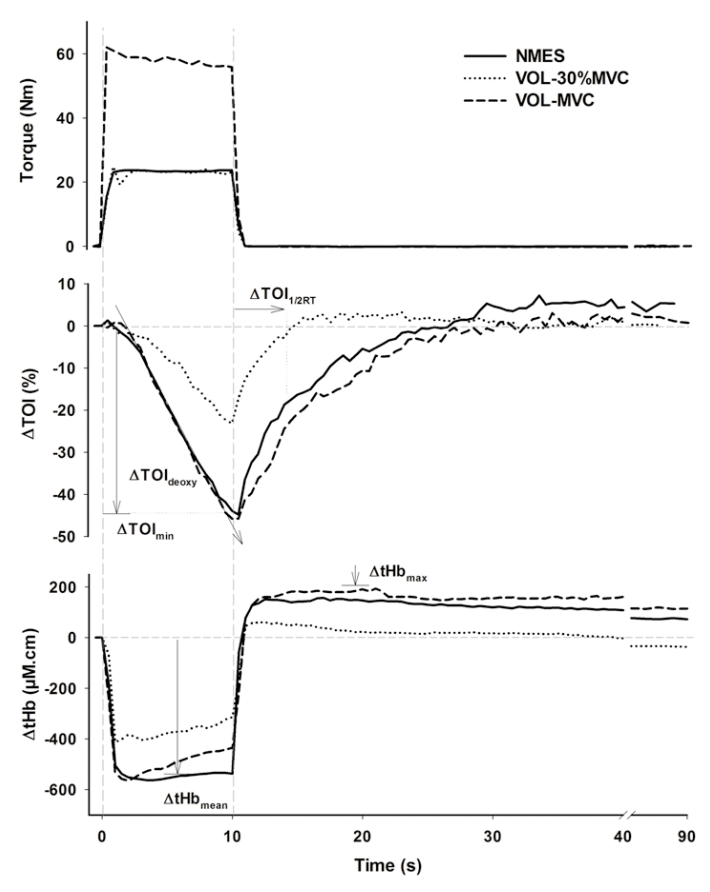

Fig 1. Typical changes in elbow flexor torque and biceps brachii tissue oxygenation index ( $\triangle T O I)$ and total hemoglobin volume $(\Delta t H b)$ during electrically evoked (NMES) and voluntary sustained isometric contractions at 30\% MVC (VOL-30\%MVC) and maximal intensity (VOL-MVC). The vertical lines represent the onset and the end of the contraction phase. $\triangle T O I_{\text {deoxy, }} \triangle T O I$ deoxygenation slope; $\triangle T O I_{\text {min }}$, minimum $\triangle T O I$ amplitude; $\triangle T O I_{1 / 2 R T}$, $\triangle T O I$ half recovery time; $\Delta t H b_{\text {mean }}$, mean $\Delta t H b$ amplitude; $\Delta t H b_{\text {max }}$, maximum $\Delta t H b$ amplitude.

conditions for the mean $30 \mathrm{~s}$ baseline TOI and $\mathrm{tHb}$ values, subsequent measurement time points were normalized as the magnitude of change from the respective baseline $\mathrm{TOI}$ and $\mathrm{tHb}$ values that was set to zero. Fig. 1 shows the determination of the NIRS parameters during and following each contraction. More specific physiological and reliability details of the respective NIRS parameters are described in our previous work. ${ }^{11,13}$

\section{Statistical Analyses}

A one-way ANOVA was used to compare torque (Torquemean and TorqueIntegral), TOI ( $\Delta$ TOIdeoxy, $\Delta$ TOImin, $\Delta$ TOI1/2RT) and tHb ( $\Delta$ tHbmean and $\Delta$ tHbmax) parameters between contraction conditions (NMES-30\%MVC, VOL-30\%MVC, and VOL-MVC). When a significant main effect (contraction condition) was found, Tukey's post-hoc tests were performed. Significance was set at $\mathrm{P}<0.05$. Statistical computations were performed using SPSS $®$ software (Lead Technologies Inc., USA). Otherwise stated, data are presented as mean \pm SEM.

\section{Results}

\section{Elbow Flexor Torque}

As shown in Fig. 1, torque increased above baseline at the onset of contraction and was maintained stable during the contraction phase for NMES and VOL$30 \%$ MVCMVC, but slightly decreased in VOL-MVC. Torquemean and TorqueIntegral were significantly $(\mathrm{P}$ $<0.001)$ lower for NMES than VOL-MVC by $\sim 65 \%$, but there was no significant difference between NMES and VOL-30\%MVC (Table 1). At the end of the contraction phase, torque quickly returned to baseline for all conditions.

\section{Tissue Oxygenation Index}

The typical changes in TOI during NMES and VOL contraction conditions are shown in Fig. 1. During the contraction phase, $\Delta$ TOIdeoxy and $\Delta$ TOImin were significantly $(\mathrm{P}=0.001)$ greater for NMES than VOL$30 \%$ MVCMVC; however, when NMES was compared to VOL-MVC, the changes in $\triangle$ TOIdeoxy and $\Delta$ TOImin were not significantly different. In the recovery period, $\Delta$ TOI returned towards baseline, such that $\Delta$ TOI1/2RT was significantly $(\mathrm{P}=0.001)$ greater for NMES than VOL-30\%MVC, but $\Delta$ TOI1/2RT was not significantly different between NMES and VOLMVC (Table 1).

\section{Total Hemoglobin Volume}

The onset of contraction provoked a rapid decrease in $\Delta \mathrm{tHb}$ from baseline and was maintained nearly constant for the duration of the contraction phase ( $\Delta$ tHbmean), although there was a slight increase in $\Delta \mathrm{tHb}$ at the end of the VOL-MVC contraction phase. The decrease in $\Delta$ tHbmean was significantly $(\mathrm{P}=0.02)$ greater for NMES than VOL-30\%MVC, but was not significantly different between NMES and VOL-MVC (Table 1). $\Delta \mathrm{tHb}$ quickly returned towards baseline immediately after the end of the contraction phase, and tHbmax was significantly $(\mathrm{P}=0.03)$ greater for NMES than VOL-30\%MVC, but was not significantly different between NMES and VOL-MVC (Table 1).

\section{Discussion}

The main findings of the study were that the NIRSderived local muscle metabolic demand during ( $\Delta$ TOIdeoxy, $\Delta$ TOImin and tHbmean) and following $(\Delta \mathrm{TOI} 1 / 2 \mathrm{RT}$ and tHbmax) a sustained (10-s) contraction was greater for NMES than VOL at the same $30 \% \mathrm{MVC}$ force level, but not different when compared to VOL at MVC force level. This finding extends previous studies ${ }^{10,14}$ by showing that NMESevoked contractions at a lower stimulation frequency $(30 \mathrm{~Hz})$ and non-maximal current intensity can induce a similar regional muscle metabolic demand as a maximal voluntary contraction.

Previous studies have documented that NMES-evoked contractions induce a greater systemic and local muscle 


\section{Muscle metabolic demand by NMES or voluntary contractions at different force levels}

Eur J Transl Myol 26 (2) 169-174

Table 1. Changes (Mean $\pm S E M, n=8$ ) in elbow flexor torque and biceps brachii tissue oxygenation index $(\triangle T O I)$ and total hemoglobin volume ( $\triangle t H b)$ parameters during Neuromuscular electrical stimulation (NMES) and voluntary sustained isometric contractions at 30\% MVC (VOL-30\%MVC) and maximal (VOL-MVC) intensity

\begin{tabular}{|l|l|l|l|}
\hline \multirow{2}{*}{} & \multicolumn{3}{|c|}{ Contraction Condition } \\
\cline { 2 - 4 } & \multicolumn{1}{|c|}{ NMES } & \multicolumn{1}{|c|}{ VOL-30\%MVC } & \multicolumn{1}{c|}{ VOL-MVC } \\
\hline Torque $_{\text {mean }}(\mathrm{Nm})$ & $22.4 \pm 1.5 \dagger$ & $22.4 \pm 1.3 \dagger$ & $64.3 \pm 4.3$ \\
\hline Torque $_{\text {Integral }}(\mathrm{Nm} . \mathrm{s})$ & $232.0 \pm 15.3 \dagger$ & $234.6 \pm 13.9 \dagger$ & $706.5 \pm 51.2$ \\
\hline $\left.\mathrm{TOI}_{\text {deoxy }} \% . \mathrm{s}-1\right)$ & $-4.5 \pm 0.4^{*}$ & $-1.8 \pm 0.4 \dagger$ & $-5.6 \pm 0.5$ \\
\hline$\Delta \mathrm{TOI}_{\min }(\%)$ & $-38.0 \pm 3.1 *$ & $-17.1 \pm 3.7 \dagger$ & $-49.4 \pm 3.3$ \\
\hline$\Delta \mathrm{TOI}_{1 / 2 \mathrm{RT}}(\mathrm{s})$ & $5.4 \pm 0.7 *$ & $2.2 \pm 0.4 \dagger$ & $3.9 \pm 0.3$ \\
\hline$\Delta \mathrm{tHb}_{\text {mean }}(\mu \mathrm{M} \bullet \mathrm{cm})$ & $-408 \pm 48^{*}$ & $-210 \pm 28 \dagger$ & $-403 \pm 46$ \\
\hline$\Delta \mathrm{tHb}_{\max }(\mu \mathrm{M} \bullet \mathrm{cm})$ & $70 \pm 5 *$ & $17 \pm 10.2 \dagger$ & $102 \pm 17$ \\
\hline
\end{tabular}

metabolic demand than VOL at the same absolute (10$50 \%$ MVC) force level4-8,15 The present study also found a greater NIRS-derived local muscle metabolic demand for NMES than VOL at the same absolute elbow flexor torque output $(30 \% \mathrm{MVC})$, such that $\triangle \mathrm{TOIdeoxy}$ and TOImin were $\sim 60 \%$ smaller for VOL-30\%MVC than NMES (Table 1). This suggests that muscle $\mathrm{O}^{2}$ demand exceeded $\mathrm{O}^{2}$ supply earlier and that the muscle $\mathrm{O}^{2}$ demand was greater for NMES than VOL at the same $30 \%$ MVC force level. During VOL-30\%MVC, the decrease in $\Delta$ tHbmean was $\sim 50 \%$ smaller than NMES (Table 1), suggesting a lower muscle blood volume displacement caused by less pressure on muscle microcirculation vessels adjacent to the NIRS probe. Furthermore, since the recovery time of TOI $(\Delta \mathrm{TOI} 1 / 2 \mathrm{RT})$ is related to muscle oxidative capacity and/or rate of blood flow/ $\mathrm{O}_{2}$ supply, ${ }^{16-18}$ the $\sim 3 \mathrm{~s}$ longer TOI1/2RT for NMES than VOL-30\%MVC would indicate that muscle $\mathrm{O}_{2}$ demand was greater than $\mathrm{O}_{2}$ supply in the early recovery period.

The main finding of the present study was that when NMES was compared to VOL at MVC force level, NIRS-derived local muscle metabolic demand was not different. These findings confirm our previous study using a similar NMES frequency $(30 \mathrm{~Hz})$ but shorter duration (1-s) repeated (30 contraction) protocol of the elbow flexors, ${ }^{15}$ and Kooistra et al. that applied NMES of the knee extensors at high frequency $(50-75 \mathrm{~Hz})$ and maximal current intensity $(70-195 \mathrm{~mA}) .{ }^{10}$ In the present study, it can be assumed that the same TOI desaturation rate $(\Delta$ TOIdeoxy) and TOI amplitude level ( $\Delta$ TOImin) attained during NMES and VOL-MVC correspond to similar local muscle $\mathrm{O}_{2}$ consumption. Furthermore, at the onset of contractions NMES and VOL-MVC resulted in a same immediate decrease in $\Delta \mathrm{tHb}$, and the mean absolute amplitude changes during the contraction phase $(\triangle \mathrm{tHbmean})$ were also not different for NMES and VOL-MVC (Fig. 1 and Table 1), which suggests that there was similar mechanical pressure on the muscle microcirculation blood vessels adjacent to NIRS probe. The same $\Delta \mathrm{TOI} 1 / 2 \mathrm{RT}$ and tHbmax in the recovery period of NMES and VOL-MVC (Fig. 1, Table 1), suggest a similar rate of increase in blood flow/ $\mathrm{O}_{2}$ supply relative to $\mathrm{O}_{2}$ demand (i.e., oxidative capacity) in the recovery period in both conditions. Overall, the NIRS-derive local muscle metabolic demand parameters during and following the sustained NMES and VOL-MVC contraction provide strong evidence that both conditions induced the same local muscle metabolic demand.

It is important to note that changes in NIRS parameters represent localized biceps brachii metabolic and hemodynamic responses, but torque is a function of the combined effect of the elbow flexor muscles. It seems likely that mainly the biceps brachii and brachioradialis were activated in NMES to produce the $30 \% \mathrm{MVC}$ torque level; however in VOL, not only the biceps brachii and brachioradialis, but also the brachialis and other synergistic muscles stabilizing the upper extremity (e.g. shoulder and back muscles) were involved in the torque production, which would account for the extra torque output for VOL-MVC than NMES.

The reason for the greater local muscle metabolic demand for NMES than VOL at the same force output has previously been suggested to be due to differences in motor unit recruitment. NMES has been described to recruit all motor units in a spatially fixed (nonselective for slow or fast motor units) and temporally synchronous motor unit activation pattern, ${ }^{3,9}$ particularly the superficial motor units adjacent to the stimulation electrodes, ${ }^{6}$ with greater levels of activation of deeper motor units with increased current intensities. ${ }^{3}$ On the other hand, VOL recruits motor units according to Henneman's size principal where progressive recruitment of slow motor units follows in order of increasing size to the fast motor units with increased intensity of VOL up to MVC level, which 


\section{Muscle metabolic demand by NMES or voluntary contractions at different force levels}

Eur J Transl Myol 26 (2) 169-174

ensures activation of both fast and slow motor units. ${ }^{3}$ Felici et al., ${ }^{19}$ using an elbow flexor NIRS experimental protocol to the present study, reported that biceps brachii metabolic demand during a voluntary 30-s sustained isometric contraction increased in proportion to elbow flexor torque (20-80\% MVC) and biceps brachii motor unit activation (assessed using EMG). Similar relationships between NIRS-derived muscle metabolic demand and EMG amplitude for the extensor carpi radialis and trapezius muscles during submaximal isometric contractions (10-70\% MVC) have been reported. ${ }^{20}$ The activation of both slow and fast motor units during sustained NMES-evoked contractions at relatively low absolute torque levels (30\%MVC) could be a reason why muscle metabolic demand was significantly greater for NMES compared to VOL$30 \% \mathrm{MVC}$ to accomplish the same submaximal absolute torque output.

These findings have relevance in understanding why NMES-evoked contractions induce a greater and faster neuromuscular fatigue, particularly when compared to VOL at the same force levels. Since the NMES current intensities required to evoke $30 \% \mathrm{MVC}$ from the biceps brachii (and brachioradialis) would continuously activate both slow and fast motor units synchronously at near maximal levels, the fatigue of these stimulated muscles is obvious following repeated contractions. It could be suggested that advanced multi channel NMES systems could be developed to share the workload between different regions of the same muscle and/or synergistic muscles throughout the stimulation period to prevent peripheral fatigue developing and to allow sufficient rest periods to allow the activated muscle fibers to resynthesize energy substrates and remove metabolites. ${ }^{21}$ The present study findings in healthy adults have clinical relevance for stroke patients and/or patients with immobilization of a joint due to injury/disuse, in which maximal voluntary contractions are limited. Gondin et al., ${ }^{22}$ have shown that an 8 week NMES training program in healthy adults induce neuromuscular adaptations similar to voluntary resistance training (i.e., muscle hypertrophy, strength gains and neural drive), as well as endurance training (i.e., a fast-to-slow shift in myosinheavy chain phenotype and glycolytic-to-oxidative shift in the metabolic profile). Clinically, it can be suggested that the inclusion of NMES during rehabilitation of disuse atrophy in hypoactive patients will improve maximal voluntary strength and activation, and may facilitate the maintenance of muscle oxidative capacity. In conclusion, the present study has shown that during a sustained isometric contraction evoked by NMES, the NIRS-derived local muscle metabolic demand (represented by $\Delta$ TOIdeoxy, $\Delta$ TOImin and tHbmean during the contraction phase and TOI1/2RT and tHbmax in the recovery period) was greater than VOL at the same absolute force level (30\%MVC), but not different to VOL at the maximal force level. Future studies are necessary to compare local muscle metabolic demand of different NMES stimulation parameters to enhance muscle force production and reduce neuromuscular fatigue.

\section{Contributions}

MM, KN: conception and design; MM, KN, SP, GK: analysis and interpretation of data; MM, KN, SP, GK: drafting the article and revising it critically for important intellectual content; MM, KN, SP, GK: final approval of the version to be published.

\section{Acknowledgements}

The authors extend their appreciation to Dr. M. Jubeau, Prof. G. Millet, Dr. N. Maffiuletti and Dr. J. Gierthmühlen for their assistance during the research project.

\section{Conflict of Interest}

The authors declare no potential conflict of interests.

\section{Corresponding Author}

Dr. Makii Muthalib, EuroMov, University of Montpellier, 700 Av. Du Pic St. Loup, Montpellier 34090, France.

E-mail: makii.muthalib@umontpellier.fr, makii.muthalib@gmail.com

E-mails of Authors

Stephane Perrey: stephane.perrey@umontpellier.fr

Kazunori Nosaka: k.nosaka@ecu.edu.au

Graham Kerr: g.kerr@qut.edu.au

\section{References}

1. Vanderthommen $M$, Duchateau J. Electrical stimulation as a modality to improve performance of the neuromuscular system. Exerc Sport Sci Rev 2007;35:180-5.

2. Bax L, Staes F, Verhagen A. Does neuromuscular electrical stimulation strengthen the quadriceps femoris? A systematic review of randomised controlled trials. Sports Med 2005;35:191-212.

3. Gregory CM, Bickel CS. Recruitment patterns in human skeletal muscle during electrical stimulation. Phys Ther 2005;85:358-64.

4. McNeil CJ, Murray BJ, Rice CL. Differential changes in muscle oxygenation between voluntary and stimulated isometric fatigue of human dorsiflexors. J Appl Physiol 2006;100:890-5.

5. Theurel J, Lepers R, Pardon L, Maffiuletti NA. Differences in cardiorespiratory and neuromuscular responses between voluntary and stimulated contractions of the quadriceps femoris muscle. Respir Physiol Neurobiol 2007;157:3417.

6. Vanderthommen M, Duteil S, Wary C, et al. A comparison of voluntary and electrically induced contractions by interleaved $1 \mathrm{H}-$ and 31P-NMRS in humans. J Appl Physiol 2003;94:1012-24. 


\section{Muscle metabolic demand by NMES or voluntary contractions at different force levels}

Eur J Transl Myol 26 (2) 169-174

7. Vanderthommen M, Depresseux JC, Bauvir P, et al. A positron emission tomography study of voluntarily and electrically contracted human quadriceps. Muscle Nerve 1997;20:505-7.

8. Jubeau M, Lef Y, Duhamel G, et al. Localized metabolic and $\mathrm{t} 2$ changes induced by voluntary and evoked contractions. Med Sci Sports Exerc 2014;47:921-30.

9. Jubeau M, Gondin J, Martin A, Sartorio A, Maffiuletti NA. Random motor unit activation by electrostimulation. Int J Sports Med 2007;28:901-4.

10. Kooistra RD, de Ruiter CJ, de Haan A. Knee angledependent oxygen consumption of human quadriceps muscles during maximal voluntary and electrically evoked contractions. Eur J Appl Physiol 2008;102:233-42.

11. Ferrari M, Muthalib M, Quaresima V. The use of near-infrared spectroscopy (NIRS) in understanding skeletal muscle physiology: recent developments. Philos Transact A Math Phys Eng Sci 2011;369:4591-4604.

12. Ferrari M, Mottola L, Quaresima V, Principles, techniques, and limitations of near infrared spectroscopy. Can J Appl Physiol 2004;29:463-87.

13. Muthalib M, Millet GY, Quaresima V, Nosaka K. Reliability of near-infrared spectroscopy for measuring biceps brachii oxygenation during sustained and repeated isometric contractions. J Biomed Opt 2010;15:017008.

14. Muthalib M, Jubeau M, Millet GY, Maffiuletti NA, Ferrari M, and Nosaka K, Biceps brachii muscle oxygenation in electrical muscle stimulation. Clin Physiol Funct Imaging 2010; 30:360-8.

15. Muthalib M, Jubeau M, Millet GY, Maffiuletti NA, Nosaka K, Comparison between electrically evoked and voluntary isometric contractions for biceps brachii muscle oxidative metabolism using nearinfrared spectroscopy. Eur J Appl Physiol 2009;107:235-41.

16. Kime R, Hamaoka T, Sako T, et al. Delayed reoxygenation after maximal isometric handgrip exercise in high oxidative capacity muscle. Eur J Appl Physiol 2003;89:34-41.

17. Kime R, Katsumura T, Hamaoka T, et al. Muscle reoxygenation rate after isometric exercise at various intensities in relation to muscle oxidative capacity. Advances in Experimental Medicine and Biology 2003;530:497-507.

18. Cettolo V, Ferrari M, Biasini V, Quaresima V. Vastus lateralis $\mathrm{O} 2$ desaturation in response to fast and short maximal contraction. Med Sci Sports Exerc 2007;39:1949-59.

19. Felici F, Quaresima V, Fattorini L, et al. Biceps brachii myoelectric and oxygenation changes during static and sinusoidal isometric exercises. J Electromyogr Kinesiol 2009;19:e1-11.

20. Elcadi GH, Forsman M, Crenshaw AG. The relationship between oxygenation and myoelectric activity in the forearm and shoulder muscles of males and females. Eur J Appl Physiol 2011;111:647-58.

21. Sayenko DG, Nguyen R, Popovic MR, Masani K. Reducing muscle fatigue during transcutaneous neuromuscular electrical stimulation by spatially and sequentially distributing electrical stimulation sources. Eur J Appl Physiol 2014;114:793-804.

22. Gondin J, Brocca L, Bellinzona E, et al. Neuromuscular electrical stimulation training induces atypical adaptations of the human skeletal muscle phenotype: a functional and proteomic analysis. J Appl Physiol 2011;110:433-50. 